\title{
Atitudes dos enfermeiros frente ao Processo de Enfermagem de um hospital público: estudo descritivo
}

\author{
Nurses' attitudes to the nursing process at a public hospital: a descriptive study \\ Actitudes de los enfermeros ante el proceso de enfermería en un hospital público: estudio descriptivo
}

\author{
Leticia Bottcher Dias', Erika Christiane Marocco Duran"
}

\begin{abstract}
RESUMO
Objetivos: descrever as atitudes dos enfermeiros de um hospital público frente ao Processo de Enfermagem (PE). Método: trata-se de um estudo descritivo. Participaram do estudo 35 enfermeiros e utilizaram-se quatro instrumentos: caracterização do sujeito, Percepções dos Enfermeiros frente ao PE, grau de conhecimento e grau de contato com o PE. Os dados foram coletados no primeiro semestre de 2016 e analisados por meio de estatística descritiva. Resultados: $O$ adjetivo menos pontuado pelos enfermeiros foi a dupla rotineiro/criativo e o mais pontuado negativo/positivo. Obteve-se também que os enfermeiros tem um baixo contato com o PE após a formação, mas avaliam ter um bom conhecimento. Conclusão: O grau de contato dos enfermeiros com o PE precisa ser ampliado para que a prática clínica possa ser aprimorada.
\end{abstract}

Descritores: Processo de enfermagem, papel do profissional de enfermagem, atitude do pessoal de saúde, administração hospitalar.

\section{ABSTRACT}

Objectives: to describe nurses' attitudes to the nursing process (NP) at a public hospital. Method: in this descriptive study, 35 nurses participated and four instruments were used to characterize the participants, their perceptions of the NP, and their knowledge of and contact with the NP. Data were collected in the first half of 2016, and analyzed using descriptive statistics. Results: the adjective least chosen by the nurses was the dual term "routine-creative", while the most chosen was "negative-positive". The nurses were also found to have little contact with the NP after graduating, but considered their knowledge to be good. Conclusion: the nurses' contact with the NP needs to be expanded so that clinical practice can be improved.

Descriptors: Nursing process, nurse's role, attitude of health personnel, hospital administration.

\section{RESUMEN}

Objetivo: describir las actitudes de los enfermeros de un hospital público ante el Proceso de Enfermería (PE). Método: se trata de un estudio descriptivo. Participaron del estudio 35 enfermeros y se utilizaron cuatro instrumentos: caracterización del sujeto, percepciones de los enfermeros ente el PE, grado de conocimiento y grado de contacto. Los datos fueron recolectados en el primer semestre de 2016 y analizados a través de estadística descriptiva. Resultados: el adjetivo menos utilizado por los enfermeros fue el dúo rutinario / creativo y el más apuntado fue negativo / positivo. También se observó que los enfermeros tienen un bajo contacto con el PE después de la universidad pero evalúan su conocimiento como siendo bueno. Conclusión: el grado de contacto de los enfermeros con el PE necesita ser ampliado para que se pueda perfeccionar la práctica clínica.

Descriptores: Proceso de enfermería, rol de la enfermera, actitud del personel de salud, administración hospitalaria.

\section{INTRODUÇÃO}

O processo de enfermagem (PE) é um instrumento metodológico que subsidia o corpo de conhecimento da enfermagem direcionando seu raciocínio, fundamentando suas ações no conhecimento técnico-científico e abrangendo os valores histórico-culturais do enfermeiro. É um instrumento sistemático que norteia o cuidado profissional, considerando os dados coletados, o julgamento sobre as respostas humanas (diagnósticos de enfermagem), o planejamento e as intervenções de enfermagem, bem como os resultados e a evolução ${ }^{1}$.
O termo Processo de Enfermagem é utilizado desde 1955, sendo considerado uma estrutura para que os cuidados fossem prestados². Mesmo já estabelecido há tanto tempo, os artigos encontrados referentes ao uso e a percepção dos enfermeiros, são escassos, divergentes e em sua maioria com mais de cinco anos da publicação ${ }^{3}$.

Associado a esse fato, a implantação do PE em hospitais é encontra empecilhos que se tornam grande dificuldade. Estudos apontam que a implementação 
do PE refere-se à imposição legal e da chefia, que exige apenas a documentação e não o uso efetivo dessa metodologia ${ }^{4-6}$. No contexto cirúrgico, também são descritas dificuldades como a não capacitação da equipe, a falta de domínio no exame físico e a escassez de recursos humanos ${ }^{1}$.

A disposição e a percepção que os enfermeiros têm para o PE refletem em atitudes pessoais de aproximação ou afastamento, o que influencia em seu comportamento $0^{4,5}$. Tais atitudes podem ser favoráveis ou desfavoráveis caracterizando uma dificuldade em implantar, manter e envolver-se no PE5. Instrumento transcultural foi desenvolvido para avaliar as atitudes dos enfermeiros frente aos diagnósticos de enfermagem e validado para o conceito de processo de enfermagem. Um estudo com o objetivo de descrever a disposição sobre o PE encontrou que a prescrição de enfermagem é pouco valorizada e é vista como rotineira, mas alerta que ela não deve ser realizada de maneira mecanizada e sem reflexão ${ }^{5}$.

O uso rotineiro e a falta de conhecimento sobre a metodologia faz com que os profissionais a utilizem de maneira incompleta, pouco acurada e não tenham conhecimento para tal ${ }^{3,7}$. Salienta-se, que as atitudes dos enfermeiros em relação ao PE influenciam em sua implementação e manutenção. Fatores pessoais que influenciam no posicionamento perante o PE são descritos como a experiência na prática clínica, a educação formal e a expectativa dos profissionais sobre si mesmos ${ }^{1}$.

As atitudes dos enfermeiros também têm grandes implicações no processo de acreditação hospitalar. A acreditação promove a qualificação dos serviços de saúde, com foco na segurança do paciente e no uso racional de instrumentos para fundamentar o cuidar, aspecto intimamente ligado ao processo de enfermagem ${ }^{8,9}$.

Diante da necessidade do uso do PE para cientificidade da profissão e da importância do posicionamento dos enfermeiros para a consolidação desta ação científica, este estudo objetivou descrever as atitudes dos enfermeiros relacionadas ao PE e o grau de conhecimento e contato destes com o PE em um hospital acreditado.

\section{MÉTOdO}

Estudo descritivo, transversal, desenvolvido em um Hospital Público de Ensino de um município do interior paulista que é certificado pela Organização Nacional de Acreditação (ONA) com o nível três, que apresenta uma excelência em gestão, motivo pela escolha desta instituição.

A instituição conta com 260 leitos e realiza consultas, internações, exames e cirurgias de sua região. A manutenção do padrão de qualidade é uma das características mais realçada do hospital.

Durante a coleta de dados, no primeiro semestre de 2016, trabalhavam na instituição 78 enfermeiros e todos foram convidados a participar do estudo, porém houve a participação 35 deles (44,87\%). Utilizou-se como instrumentos de pesquisa:

- Caracterização da população: dados pessoais e do trabalho

- Grau de conhecimento sobre o Processo de Enfermagem - o participante escolhia entre nenhum, pouco, moderado ou muito o seu conhecimento sobre o PE e suas fases ${ }^{1}$.

- Grau de contato com o Processo de Enfermagem - o participante escolhia entre nenhum, pouco, moderado ou muito o seu contato com o PE por meio de realização de leitura, participação em aulas/cursos, participação em eventos, uso na prática clínica e realização de pesquisa ${ }^{1}$.

- Posições sobre o Processo de Enfermagem (PPE) composto por 20 duplas de adjetivos que estão separados por uma escala de sete pontos equidistantes e o sujeito é convidado a assinalar a proximidade que tem com 0 adjetivo em relação do Processo de Enfermagem ${ }^{1,5}$.

O instrumento de Posições sobre o Processo de Enfermagem avaliou as atitudes dos enfermeiros frente ao PE. A disposição favorável e a disposição desfavorável estavam separados por uma escala semântica de 1 a 7 e para cada adjetivo quanto mais elevado o número, mais favorável a atitude. Para apresentação dos resultados, foram realizadas as médias das respostas e os adjetivos que estavam invertidos no instrumento foram corrigidos para análise ${ }^{1,5}$.

Os dados foram analisados por meio do software estatístico SAS versão 9.4 e descritos nas tabelas abaixo.

\section{RESULTADOS}

Na amostra, $85,71 \%(n=30)$ eram do sexo feminino, a idade variou de 26 a 49 anos, em média 35,4 (DP $\pm 6,2$ ). Do total de enfermeiros $51,43 \%$ referiram ter cursado especialização (Latu sensu), 2,86\% mestrado (Stricto sensu) e 45,71\% referiram não ter cursado pósgraduação. O tempo médio de trabalho na instituição é de cinco anos e 11 meses, sendo o mínimo um mês e o máximo 14 anos e nove meses. Quanto ao tempo de formado, a média é de 10 anos e um mês, sendo o mínimo cinco anos e o máximo 28 anos.

A distribuição de enfermeiros segundo suas funções mostra que a maioria dos enfermeiros não exercia cargo de chefia ( $80 \%)$ e tinham como principal atividade o planejamento de cuidados (48,57\%), seguido de atividades administrativas $(25,71 \%)$ e cuidado direto $(22,86 \%)$. Eram enfermeiros que trabalhavam em sua unidade de preferência (74,29\%); estavam totalmente satisfeitos com o trabalho $(57,14 \%)$ e estavam satisfeitos com sua escolha profissional (68,57\%). Foram contemplados enfermeiros de todos os turnos de trabalho manhã, tarde, diurno, noturno.

A tabela 1 apresenta os resultados do instrumento de grau de contato e grau de conhecimento com o PE. 
Tabela 1: Grau de conhecimento e de contato com o PE. Sumaré, São Paulo, 2016

\begin{tabular}{|c|c|c|}
\hline Variável & $\mathrm{n}$ & $\%$ \\
\hline \multicolumn{3}{|l|}{ GRAU DE CONHECIMENTO } \\
\hline \multicolumn{3}{|l|}{ PE em geral } \\
\hline Moderado & 22 & 62,86 \\
\hline Muito & 13 & 37,14 \\
\hline \multicolumn{3}{|l|}{ Entrevista e exame físico } \\
\hline Moderado & 16 & 45,71 \\
\hline Muito & 19 & 54,29 \\
\hline \multicolumn{3}{|c|}{ Diagnóstico de enfermagem } \\
\hline Moderado & 23 & 65,71 \\
\hline Muito & 12 & 34,29 \\
\hline \multicolumn{3}{|c|}{ Prescrição de enfermagem } \\
\hline Moderado & 19 & 54,29 \\
\hline Muito & 16 & 45,71 \\
\hline \multicolumn{3}{|l|}{ Evolução de enfermagem } \\
\hline Pouco & 1 & 2,86 \\
\hline Moderado & 20 & 57,14 \\
\hline Muito & 14 & 40,00 \\
\hline \multicolumn{3}{|l|}{ GRAU DE CONTATO } \\
\hline \multicolumn{3}{|l|}{ Realização de leitura } \\
\hline Nenhum & 5 & 14,29 \\
\hline Pouco & 5 & 14,29 \\
\hline Moderado & 15 & 42,86 \\
\hline Muito & 10 & 28,57 \\
\hline \multicolumn{3}{|c|}{ Participação em aulas/cursos } \\
\hline Nenhum & 4 & 11,43 \\
\hline Pouco & 8 & 22,86 \\
\hline Moderado & 21 & 60,00 \\
\hline Muito & 2 & 5,71 \\
\hline \multicolumn{3}{|l|}{ Participação em eventos } \\
\hline Nenhum & 7 & 20,00 \\
\hline Pouco & 12 & 34,29 \\
\hline Moderado & 14 & 40,00 \\
\hline Muito & 2 & 5,71 \\
\hline \multicolumn{3}{|l|}{ Uso na prática clínica } \\
\hline Nenhum & 2 & 5,71 \\
\hline Pouco & 2 & 5,71 \\
\hline Moderado & 17 & 48,57 \\
\hline Muito & 14 & 40,00 \\
\hline \multicolumn{3}{|l|}{ Realização de pesquisa } \\
\hline Nenhum & 9 & 25,71 \\
\hline Pouco & 16 & 45,71 \\
\hline Moderado & 10 & 28,57 \\
\hline
\end{tabular}

Com relação ao grau de conhecimento do $\mathrm{PE}$, $62,68 \%(n=22)$ dos enfermeiros relatam conhecer moderadamente o PE em geral e $37,14 \%(n=13)$ relatam conhecer muito. $\mathrm{O}$ grau de contato foi classificado, em sua maior parte das vezes, como moderado, porém as respostas pouco e nenhum foram significativas quando comparado ao grau de conhecimento.

Os resultados do instrumento Posições sobre o Processo de Enfermagem estão descritos na tabela 2.
Tabela 2: Estatística dos escores dos itens do PPE. Sumaré, São Paulo, 2016.

\begin{tabular}{lc}
\hline Itens PPE & Média (₫DP) \\
\hline Rotineiro/criativo & $4,40( \pm 2,26)$ \\
Difícil/fácil & $4,60( \pm 1,77)$ \\
Desagradável/agradável & $5,43( \pm 1,65)$ \\
Dificultador/facilitador & $5,71( \pm 1,62)$ \\
Fraco/forte & $5,77( \pm 1,33)$ \\
Desconfortável/confortável & $5,83( \pm 1,40)$ \\
Não recompensador/recompensador & $5,91( \pm 1,65)$ \\
Ambíguo/claro & $6,03( \pm 1,12)$ \\
Não realista/realista & $6,06( \pm 1,03)$ \\
Inconveniente/conveniente & $6,11( \pm 1,11)$ \\
Inaceitável/aceitável & $6,37( \pm 0,94)$ \\
Não significativo/significativo & $6,43( \pm 0,88)$ \\
Bobo/inteligente & $6,43( \pm 0,78)$ \\
Inválido/válido & $6,43( \pm 0,74)$ \\
Ruim/bom & $6,43( \pm 0,92)$ \\
Sem valor/valioso & $6,46( \pm 1,12)$ \\
Irrelevante/relevante & $6,46( \pm 0,89)$ \\
Insignificante/significante & $6,51( \pm 0,78)$ \\
Sem importância/importante & $6,51( \pm 0,85)$ \\
Negativo/positivo & $6,57( \pm 0,70)$ \\
\hline Total & 6,02 \\
\hline
\end{tabular}

Quanto maior a média, mais favorável o enfermeiro foi em relação à dupla de adjetivos. O escore médio mais baixo nos itens do PPE foram para as duplas rotineiro/criativo $(4,4)$ e difícil/fácil $(4,60)$ e os mais elevados para negativo/positivo $(6,57)$ e sem importância/ importante $(6,51)$.

\section{DISCUSSÃo}

Mantendo o quadro encontrado nos estudos na área, a maior parte da população de enfermeiros é de sexo feminino e cerca de metade tem algum tipo de pós-graduação, o que alinha as necessidades atuais do país com a prática profissional ${ }^{5,8}$. A formação dos profissionais se deu, em média, há dez anos. Pode-se considerar que praticamente a totalidade já teve em sua formação básica a introdução do estudo do PE, o que aumenta o grau de conhecimento frente ao $P E^{9,10}$. Estudos apontam que a maioria dos conhecimentos, habilidades e competências do perfil profissional são constituídos a partir da formação na graduação, o que condiz com os dados encontrados ${ }^{10,11}$.

A maioria dos enfermeiros apresentou aprimoramento profissional com a realização de cursos Strictu sensu e Latu sensu. Salienta-se que a pós-graduação figura como impulsionadora da ciência, da tecnologia e da inovação, particularmente nesse estudo, no contexto da enfermagem. Esse fato incrementa a cientificidade da prática da enfermagem brasileira que se traduz no cuidado eficiente e eficaz ao indivíduo, família e comunidade e ensino de saúde com qualidade ${ }^{12}$. 
Discute-se que para uma assistência com excelência fazem parte das competências necessárias o envolvimento do enfermeiro com o cuidado direto, estando próximo do processo de hospitalização, ao contrário do encontrado no estudo que, por mais que se trate de um hospital acreditado, apenas $22,86 \%$ ( $n=8)$ dos enfermeiros responderam realizar cuidados diretos ao paciente na maior parte do tempo, mesmo $80 \%$ da amostra não exercendo cargo de chefia ${ }^{13}$.

O grau de conhecimento do PE está mais relacionado à entrevista e exame físico, parte prática, do que a parte teórica de diagnóstico, prescrição e evolução. Ambas as partes tiveram altas pontuações de conhecimento, o que significa que os enfermeiros realizam o PE com embasamento clínico, mas reconhecem a necessidade de sustentação teórica. Salienta-se que os enfermeiros conhecem o PE, entretanto, apresentam pouco contato com essa ferramenta metodológica. Desta forma, fazse necessário o aprofundamento em estudos, seja por meio de cursos, capacitações ou educação continuada, de maneira a subsidiar os enfermeiros a realizar o PE de maneira reflexiva e com aprofundamento, o que oferece qualidade ao cuidado ${ }^{3,5}$.

O número de respostas pouco e nenhum no instrumento de grau de contato confirmou o encontrado em outros estudos que relaciona as atividades acerca do PE na maior parte das vezes com a graduação e dificilmente após a formação. Sabe-se que a pós-graduação eleva o nível do cuidado prestado, assim faz-se necessário que os programas de especialização, mestrados e doutorados abordem o PE e aprimorem tal prática clínica ${ }^{5,10}$.

Referente à pontuação media nos itens do PPE, a menor media foi a da dupla de adjetivos rotineiro/ criativo $(4,4)$. É indicado pela literatura analisar os itens com escore $\geq 5,5$ como fortemente favoráveis; e aqueles com escore médio $\leq 4,5$ como mais desfavoráveis ${ }^{14}$.

Frente ao cenário, infere-se que a realização do PE diariamente configura-se com um fazer que não instiga o pensamento crítico e a reflexão ou não os propicia, sendo necessário desenvolver ferramentas, impressos e tecnologias que tornem sua execução mais fácil e agradável já que visa uma assistência de melhor qualidade 5 .

Os adjetivos mais pontuados no instrumento PPE foram a insignificante/significante, sem importância/ importante e negativo/positivo com média acima de 6,5 . Tal resultado demonstra uma posição favorável dos enfermeiros frente manutenção e aprimoramento do $P E$, o que facilita a sua execução e desenvolvimento. Esta atitude também é exemplificada em estudos que identificam as percepções, apontando que os enfermeiros consideram que o PE beneficia o paciente e dá condições para cientificidade ${ }^{1,5,7,15}$.

Um estudo com o objetivo de conhecer os desafios enfrentados por enfermeiros para implementação do PE e possíveis estratégias encontrou que os fatores encontrados como impedimentos à implantação são os valores dos enfermeiros, a dinâmica de trabalho, a falta de apoio da instituição, a falta de enfermeiros e a falta de experiência com o método ${ }^{16}$. Encontrou-se no estudo que os enfermeiros têm atitudes favoráveis, portanto, faz-se necessário que os hospitais se apoiem em estratégias como o apoio da gestão, a promoção de práticas pedagógicas e a consideração do PE para o dimensionamento adequado ${ }^{16,17}$. É encorajada uma reorganização institucional que deve alinhar essa necessidade com a educação continuada nos hospitais ${ }^{5,7}$.

Experiências de implantação e envolvimento de enfermeiros têm sido descritas com sucesso a partir de reuniões para capacitação do $\mathrm{PE}$, semiologia e exames laboratoriais e adoção de referenciais para o serviço ${ }^{18}$.

É imprescindível que os enfermeiros reconheçam as suas limitações no que se referem ao conhecimento da práxis do $\mathrm{PE}$, por meio da identificação das dificuldades apresentadas durante o raciocínio clínico ou processo de diagnosticar e do registro das etapas dessa ferramenta metodológica para que a implantação de uma assistência de qualidade e documentada seja factível.

\section{CONCLUSÃo}

O PE encontra-se em aperfeiçoamento no Brasil, especialmente, neste estudo, em um hospital acreditado com nível de excelência. É importante que as instituições se preocupem com a sua realização, não apenas pelo aspecto legal, mas também para o incremento e a melhora da qualidade de assistência e consequentemente, da qualidade de vida do paciente.

Os profissionais apresentam uma atitude positiva frente ao $P E$, porém, precisam de incentivo para manter suas práticas além do período de formação, estímulo para melhorar o raciocínio clínico e a documentação envolvidos no PE e serem valorizados em relação a esta prática, acreditando e visualizando a mudança que seu acurado uso proporciona. Os hospitais devem criar grupos de estudos e de aperfeiçoamento que associados à atitude favorável elevará o nível do $\mathrm{PE}$, trazendo qualidade e visibilidade à enfermagem. Novas pesquisas devem ser realizadas com o objetivo de compartilhar experiências de sucesso nas intervenções em serviços e qualificação aos profissionais.

\section{REFERÊNCIAS}

1. Ribeiro E, Ferraz KMC, Duran ECM. Atitudes dos enfermeiros de centro cirúrgico diante da sistematização da assistência de enfermagem perioperatória. Rev SOBECC [Internet]. 2017; 22(4):201-7. Doi: https://doi.org/10.5327/Z1414-4425201700040005

2. Zamanzadeh V, Valizadeh L, Tabrizi FJ, Behshid M, Lotfi M. Challenges associated with the implementation of the nursing process: A systematic review. Iranian Journal of Nursing and Midwifery Research[Internet]. 2015; 20(4):411-19. Doi: 10.4103/17359066.161002 
3. Boaventura AP, Santos PA, Duran ECM. Conhecimento teórico-prático do Enfermeiro sobre Processo de Enfermagem e Sistematização de Enfermagem. Enfermería Global [Internet]. 2017;46:194205. Doi: http://dx.doi.org/10.6018/eglobal.16.2.247911

4. Marinello NP, Silva ARA, Silva DNO. Nursing care systematization - challenges for its implementation. Revista Enfermagem Contemporânea [Internet]. 2015; 4(2):254-63. Doi: http://dx.doi. org/10.17267/2317-3378rec.v4i2.523

5. Guedes ES, Turrini RNT, Sousa RMC, Baltar VT, Cruz DALM. Atitudes dos profissionais de enfermagem relacionadas ao Processo de Enfermagem. Rev. Esc. Enferm. USP [Internet]. 2012; 46,:1307. Doi: http://dx.doi.org/10.1590/S0080-62342012000700019

6. Conselho Federal de Enfermagem (BR). Resolução COFEN n.358, de 15 de outubro de 2009: dispõe sobre a sistematização da assistência de enfermagem - SAE nas instituições de saúde brasileiras. Brasília; 2009

7. Diniz IA, Cavalcante RB, Otoni A, Mata LRF. Perception of primary healthcare management nurses on the nursing process. Rev. bras. enferm. [Internet]. 2015 68(2):206-13. Doi: http://dx.doi. org/10.1590/0034-7167.2015680204i

8. Marcelino CF, Alves DFS, Guirardello EB. Autonomia e controle do ambiente de trabalho por profissionais de Enfermagem reduzem índices de exaustão emocional. REME rev. min. enferm. 2018; 22:e-1101. Doi: http://www.dx.doi.org/10.5935/14152762.20180029

9. Duran ECM, Toledo VP. Análise da produção do conhecimento em processo de enfermagem: estudo exploratório-descritivo. Rev. gaúch. enferm. [Internet]. 2011; 32(2):234-40. Doi: http://dx.doi. org/10.1590/S1983-14472011000200004

10.Santos ADB, Oliveira KKD, Rosário SSD, Lira ALBC, Tourinho FSV, Santos VEP. Estratégias de ensino-aprendizagem do processo de enfermagem na graduação e pós-graduação de enfermagem. J. res.: fundam. care. Online[Internet]. 2013; 6(3):1212-20. Doi: 10.9789/2175-5361.2014v6n3p1212
11. Pires AS, Souza NVDO, Penna LHG, Tavares KFA, D’oliveira CAFB, Almeida CM. A formação de enfermagem na graduação: uma revisão integrativa da literatura. Rev enferm UERJ [Internet]. 2014; 22(5):705-11. http://dx.doi.org/10.12957/reuerj.2014.11206

12.Scochi CGS, Munari DB, Gelbcke FL, Ferreira MA. Desafios e estratégias dos programas de pós-graduação em enfermagem para a difusão da produção científica em periódicos internacionais. Esc. Anna Nery [Internet]. 2014;18(1):5-10. Doi: 10.5935/14148145.20140001

13. Luvisotto MM, Vasconcelos AC, Sciarpa LC, Carvalho R. Patient care and administrative activities of nurses in clinical/surgical units. Einstein [Internet]. 2010; 8(2):209-14. Doi: http://dx.doi. org/10.1590/s1679-45082010ao1354

14. Lunney M, Krenz MA. An instrument to measure attitudes toward nursing diagnosis. In: Carrol-Johnson RM, Paquette $M$, eds. Classification of Nursing Diagnoses Proceedings of the Tenth Conference of North American Nursing Diagnosis Association. San Diego: Lippincott; 1992. p.389-90

15. Oliveira CM, Carvalho DV, Peixoto ERM, Camelo LV, Salviano MEM. Percepção da equipe de enfermagem sobre a implementação do processo de enfermagem em uma unidade de um hospital universitário. REME rev. min. enferm. 2012; 16(2):258-63.

16. Trindade LR, Ferreira AM, Silveira A, Rocha EM. Processo de enfermagem:desafios e estratégias para sua implementação sob a ótica de enfermeiros. Rev Santa Maria [Internet]. 2016; 42(1):7582. Doi: http://dx.doi.org/10.5902/2236583419805

17. Kirchesch CL. Strategies to implement the systematization of nursing care in the health services: an integrative review. Rev. Interd. 2016; 9(4):173-80.

18. Lopes MHBM. Experiência de implantação do processo de enfermagem utilizando os diagnósticos de enfermagem (taxonomia da Nanda), resultados esperados, intervenções e problemas colaborativos. Rev. latinoam. enferm. [Internet]. 2000; 8(3):115-8. Doi: http://dx.doi.org/10.1590/S0104-11692000000300017 